\title{
Three Zone Asymmetric Pupil Filter for Increasing Two-point Resolution of Optical Imaging Systems
}

\author{
Andra N. K. Reddy ${ }^{1,2, *}$ and Dasari K. Sagar ${ }^{2}$ \\ ${ }^{I}$ Department of Physics ( $H \& S$ ), CMR Institute of Technology, Kandlakoya (v), Medchal Road, Ranga Reddy, Hydera- \\ bad- 501 401, Telangana, India \\ ${ }^{2}$ Optics Research Group, Department of Physics, University College of Science, Osmania University, Hyderabad- \\ 500007, Telangana, India
}

\begin{abstract}
In the present study, influence of three zone asymmetric apodizer on the resolution of composite image of two object points with given intensity ratio 'c' has been investigated. The proposed pupil function and its transmittance is formulated and studied. Fourier analytical properties of three zone aperture systems are studied by applying the modified sparrow criterion. The results are discussed in terms of sparrow limits obtained as a function of the degree of coherence of the illumination, intensity ratio of two object points and apodization parameters. Additionally, a test for the resolvability of the point sources from various set of observations is presented. It is established that for certain cases of illumination, the presence of amplitude and phase apodizer leads to an improvement in the resolution of optical imaging systems. The dependence of these characteristics on apodization controlling parameters is studied and reported.
\end{abstract}

Keywords: Asymmetric apodization, Two-point resolution, sparrow resolution limit, three zone pupil filter, diffraction.

\section{INTRODUCTION}

In imaging science, resolution is always an important classical problem. Since it is not explicitly defined, it is interpreted in many ways. Current paper work examines the concept of two-point resolution. Using pupil filters the twopoint resolution of optical imaging system can be altered such that the resolution limit increases as the degree of coherence increases. Technically feasible applications for this consequence include e.g. superresolution or increased instrumental light shaping, detecting the direct image of fading object in the close proximity of the bright object. Earlier studies in this direction are limited to a few cases [1-17]. In many past approaches, images were calculated mathematically thus so-called classical resolution criteria, such as Rayleigh criterion [18]. It provides resolution limits that are exclusively dogged by the calculated shape of the PSF associated with the imaging aperture and the wavelength of incident radiation. But in real practice, such images do not occur.

Here we investigate the effect of three zone asymmetric apodizer on two-point resolution by utilizing Sparrow criterion. Two object points can just be resolved when the second derivative of the total intensity distribution in the composite image is zero [19]. The influence of defocusing on the resolution of rotationally symmetric optical systems with shaded apertures was studied extensively [20]. These studies are

*Address correspondence to this author at the Department of Physics ( $\mathrm{H} \&$ S), CMR Institute of Technology, Kandlakoya (v), Medchal Road, Ranga Reddy, Hyderabad- 501 401, Telangana, India; Tel: 09866802424;

E-mail: naarereddy@gmail.com basis for present investigation. It is clear that the current study is dealing with an effect of three zone amplitude and phase filter on resolution of asymmetrically apodized optical systems for two object points in close proximity. The axial spatial resolution of imaging system depends on the sum of the numerical apertures of illumination, incident wave front and the imaging optics [21].

The concept of asymmetric apodization introduced by Cheng and Siu [22] and succeeded in improving the resolution of point spread function. In extension to their work [23] they succeeded in achieving low optical sidelobes and sharp central maxima on one side of the PSF termed as 'good side' at the cost of aggravating its counterpart termed as 'bad side'. Studies on asymmetric apodization are found to be important in fields like confocal imaging [24-26], medical imaging, spectroscopy, communication engineering and astronomical observations [27]. This paper is organized as follows: in section 2, we briefly review the formulation and the transmittance of the pupil function and its numerical simulation is also described by employing the optimized method of numerical integration. In section 3 , we discussed the applicability of the proposed apertures for improving the resolution of optical systems. Section 4 is forming conclusions of the study.

\section{THEORY}

Considering two distinct self-luminous point sources can be imaged by two-dimensional diffraction limited imaging systems. According to geometrical optics each individual is not imaged as a point itself due to the diffraction at the aperture. According to the wave theory of fraunhofer diffraction, 


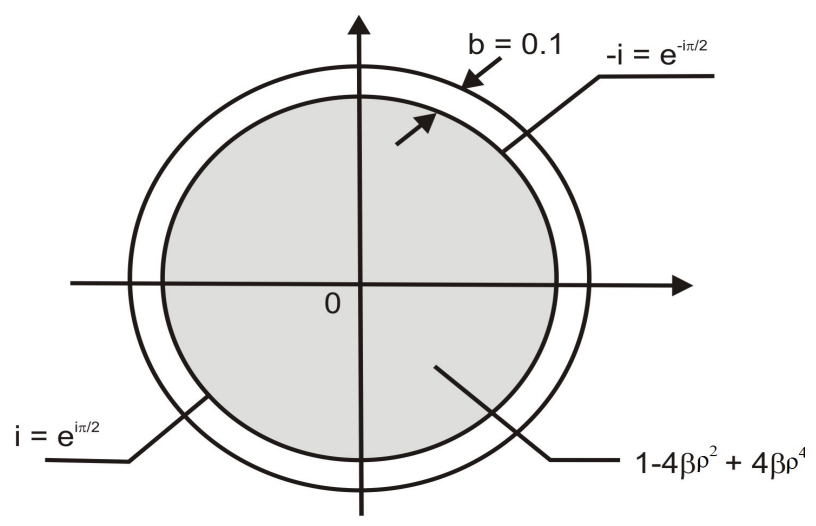

Fig. (1). General scheme of the two-dimensional three-zone aperture.

the composite intensity distribution in the image plane produced by two point sources together is given by [20] \& [21]:

$$
\begin{aligned}
& \mathrm{I}(\mathrm{X})=|\mathrm{A}(\mathrm{X}-\mathrm{B})|^{2}+\mathrm{c} \mid\left(\left.\mathrm{A}(\mathrm{X}+\mathrm{B})\right|^{2}+2 \sqrt{c} \gamma\left(\mathrm{X}_{0}\right) \mid \mathrm{A}(\mathrm{X}-\right. \\
& \mathrm{B}) \| \mathrm{A}(\mathrm{X}+\mathrm{B}) \mid
\end{aligned}
$$

Where $2 \mathrm{~B}=\mathrm{X}_{0}$ is the actual severance between the point sources, $c$ is the ratio of their intensities and $\gamma\left(X_{0}\right)$ is the true part of the complex degree of spatial coherence of the illumination. $\mathrm{X}$ is the dimension less diffraction co-ordinate in the image plane. $\mathrm{A}(\mathrm{X}+\mathrm{B})$ and $\mathrm{A}(\mathrm{X}-\mathrm{B})$ are the normalized complex amplitude whim response functions of the optical imaging system corresponding to the point sources, each of which is located at a distance of $\mathrm{X}_{0} / 2$ on both side of the optical axis. The amplitude impulse response function A (X $\pm \mathrm{B})$ is known by:

$$
A(X \pm B)=2 \int_{0}^{1} f(\rho) J_{0}[(X \pm B) \rho] \rho d \rho
$$

$$
f(\rho)=\left(1-4 \beta \rho^{2}+4 \beta \rho^{4}\right)
$$

Where $\mathrm{J}_{0}$ is the Bessel function of the first kind and zero order [20], $f(\rho)$ sets for the pupil function, here ' $\rho$ ' is the distance of the reference point on the exit pupil uttered as a fraction of the radius of the pupil and $\beta$ is the amplitude apodization parameter which decides the degree of nonuniformity of transmission of central circular region of the pupil function. The spatial distribution of transmittance in the plane containing exit aperture referred as the pupil function. Hence, the generalized expression for amplitude impulse response is given by $[22,23]$ :

$$
\begin{aligned}
& A(X \pm B)=\int_{0}^{-\delta}\left[\int_{0}^{1-b} f(\rho) J_{0}(X \pm B) \rho d \rho\right] \\
& X d X+i \int_{1-b}^{1} \int_{\frac{\pi}{2}}^{\frac{3 \pi}{2}} \exp (i x \rho \cos (\varphi-\phi)) \rho d \rho d \phi-i \\
& \int_{1-b}^{1} \int_{-\frac{\pi}{2}}^{\frac{\pi}{2}} \exp (i x \rho \cos (\varphi-\phi)) \rho d \rho d \phi
\end{aligned}
$$

Taking into consideration the requirement for resolving the composite image of two object points in close proximity with given intensity ratio 'c', we propose the twodimensional complemetary pupil function. In the present context the word 'composite image' refers to an overlapped pattern of visible light of photons collected in the focal plane. Fig. (1) shows model of the complemetary pupil functions. It consists of two semicircular edge zones of equal width ' $b$ ' with opposite phase transmittances of the form $+i$ and $-\mathrm{i}$ and the central circular zone is being amplitude apodized and its corresponding phase is zero. For proposed amplitude apodizer, the transmittance at the center of the aperture $(\rho=0) t(\rho)=$ unity i.e. maximum. It decreases towards the edge of the aperture as $\rho$ goes to 0.5 for all values of $\beta$. It is clear that $t(\rho)$ is minimum at edges for any degree of amplitude apodization. $\beta$ is the apodization parameter controlling the degree of non-uniformity of the transmittance over zone of radius (1-b). The range of values it takes is $0 \leq \beta \leq 1$. It is clear that for $\beta=0$, the transmittance of this zone is uniform. Transmittance over rest of the two zones of circular aperture of unit radius is unity. Here $b$ is the certain width of semi-circular ring. It is illustrated in Fig. (2) in detail.

In the presence of three zone apodizer the intensity distribution in the image plane is derived by combining Eq. 4 into Eq. (1). When the second derivative of the image intensity distribution vanishes at a certain point $\left(x=x_{0}{ }^{1}\right)$ between two Gaussian image points, with the condition that this point $x_{0}{ }^{1}$ should be a solution for the first derivative of the image intensity distribution becoming zero. It can be written as:

$$
\begin{gathered}
\left|\frac{\partial^{2} I(x)}{\partial x^{2}}\right|_{x=x_{0}^{1}}=0 \\
\left|\frac{\partial I(x)}{\partial x^{2}}\right|_{x=x_{0}^{1}}=0
\end{gathered}
$$

\section{RESULTS AND DISCUSSION}

In the present paper the resolution of composite image of two point sources has been evaluated by employing a 


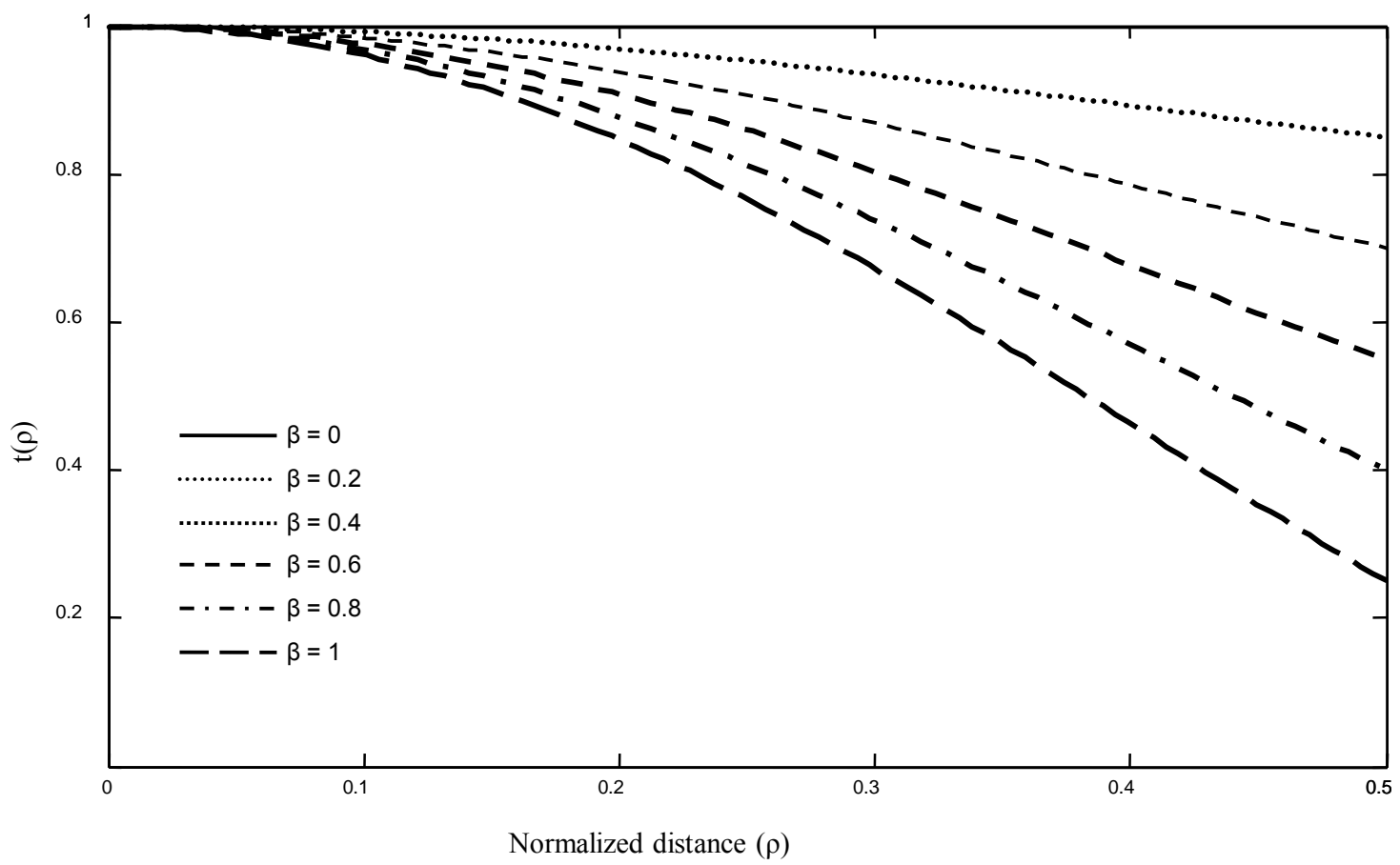

Fig. (2). Decrease of amplitude transmittance $t(\rho)$ with the normalized distance $(\rho)$.

twelve-point Gauss quadrature numerical method of integration. It has been applied to find the sparrow limits of resolution for various set of observations, are tabulated and presented. These values are obtained for the Airy case $(b=0$, $\beta=0)$ and the non-Airy case $(b \neq 0, \beta \neq 0)$. It has been found that the results of Airy case count with apodized case (threezone aperture) to better understand the efficiency of apodization. By employing Eq. (1) the intensity distribution in the composite image of the two-point sources formed by a complemetary pupil function of optical imaging system has been computed. Study has done as functions of various parameters: width of semicircular edge ring element $b$, amplitude apodization controlling parameter $\beta$, the intensity ratio of two point sources $\mathrm{c}$, the degree of coherence of illumination $\gamma$ and the actual distance of separation between two points Xo.

With the aim to increase the resolution we designed three zone apodizer by adopting the procedure as mentioned in the section 2. For composite intensity distribution of two distinct point sources with $\mathrm{c}=0.2$, the sparrow limit decreases almost linearly with the increase of the semicircular edge ring width $b(>0.06)$ for zero amplitude apodization $(\beta=0)$ under any condition of coherence of illumination. Analogous trend is found for $\beta=0.2$ under same conditions. This can be seen in more detail from the listed values in Table 1. This study clearly manifests that initially the resolution of two object points is increased as the $b$ value approaches 0.04 for all coherence conditions of illumination. On further rise in $b$ value the resolution is degraded.

For $c=0.4$ and $\beta=0$, initially the sparrow resolution limit increases as $b$ increases from 0 to 0.04 and then decreases with $b$ values under incoherent illumination. For incoherent illumination the sparrow limits are 3.6982 (Airy Case), $3.8629,3.9373,3.8936,3.7706$ and 3.6290. Similar trend is noticed for $\beta=0.2,0.4$ and 0.6 , where as in the case of coherent illumination the sparrow limits are 5.1084 (Airy Case), 5.2528, 5.1153, 4.7319, 4.3824 and 4.1778, respectively. It specifies that initially modified sparrow limit increases with semicircular edge ring width $b$ and then after exhibits a decreasing trend. In this case sparrow limits are obtained than that of unapodized case (Airy). This effect is further improved by degree of amplitude apodization in the central region of the complemetary pupil function for all $b$ values under different coherence conditions of illumination for composite image of two distinct point sources. This is clearly depicted as listed values in Table 1. It is an important aspect of present study depict that by employing amplitude and phase apodization the overall resolution of two unequally bright points is improved in terms of the modified sparrow criterion. This influence appears in the resultant image intensity distribution as presented in the following Figs. (3 and 4).

Fig. (3) shows the effect of amplitude apodization in the resolution of two point sources of different intensity at $\mathrm{Xo}=$ 4 under incoherent illumination. It is found that the overall resolution is improved with degree of amplitude apodization. In this case the critical sparrow limits found to be 3.5168 , 3.5868, 3.6820, and 3.5279 as shown in Table $\mathbf{1}$.

Fig. (4) shows the effect of amplitude-phase apodization in the resolution of incoherent composite image of two object points with given intensity ratio ' $c$ ' at $\mathrm{Xo}=4$. For $\mathrm{b}=$ 0.04 , it is observed that there is a shift found in the resultant pattern for all $\beta$ values, in addition the left half axis is becoming broadened at the cost of narrow intensity profile on the right half axis. The sparrow limits found in this case are 
Table 1. Shows the sparrow limits of resolution as a function of $b$ and $\beta$ values for a various set of values of intensity ratio $c$ and degree of coherence $\gamma$.

\begin{tabular}{|c|c|c|c|c|c|c|c|c|c|c|c|c|c|}
\hline \multicolumn{2}{|c|}{$\boldsymbol{\beta}=\mathbf{0}$} & \multicolumn{6}{|c|}{ Sparrow Limits (Xo) } & \multirow{2}{*}{$\begin{array}{c}\beta=0.2 \\
b=0\end{array}$} & \multicolumn{5}{|c|}{ Sparrow Limits (Xo) } \\
\hline c & $\gamma$ & $\mathbf{b}=\mathbf{0}$ & 0.02 & 0.04 & 0.06 & 0.08 & 0.1 & & 0.02 & 0.04 & 0.06 & 0.08 & 0.1 \\
\hline \multirow{6}{*}{0.2} & 0 & 3.8295 & 4.1216 & 4.3341 & 4.4011 & 4.3089 & 3.1441 & 3.9407 & 4.2535 & 4.4441 & 4.4406 & 3.3487 & 3.2030 \\
\hline & 0.2 & 4.2047 & 4.4670 & 4.6328 & 4.6383 & 4.4873 & 3.4839 & 4.3140 & 4.5918 & 4.7261 & 4.6497 & 4.4245 & 3.4876 \\
\hline & 0.4 & 4.5487 & 4.7795 & 4.9010 & 4.8438 & 4.6323 & 3.8585 & 4.6547 & 4.8949 & 4.9748 & 4.8250 & 4.5441 & 3.7826 \\
\hline & 0.6 & 4.8596 & 5.0641 & 5.1454 & 5.0246 & 4.7504 & 4.2944 & 4.9570 & 5.1692 & 5.2001 & 4.9757 & 4.6394 & 4.0854 \\
\hline & 0.8 & 5.0917 & 5.3070 & 5.3708 & 5.1849 & 4.8503 & 4.5404 & 5.1787 & 5.4104 & 5.4073 & 5.1030 & 4.7166 & 4.3859 \\
\hline & 1 & 5.1109 & 5.4731 & 5.5766 & 5.3271 & 4.9336 & 4.5989 & 5.2144 & 5.5937 & 5.6003 & 5.2136 & 4.7815 & 4.4614 \\
\hline \multirow{6}{*}{0.4} & 0 & 3.6972 & 3.8629 & 3.9373 & 3.8936 & 3.7706 & 3.6290 & 3.7809 & 3.9488 & 3.9882 & 3.8904 & 3.7314 & 3.4559 \\
\hline & 0.2 & 4.0454 & 4.1847 & 4.2165 & 4.1212 & 3.9548 & 3.7900 & 4.1302 & 4.2659 & 4.2536 & 4.0963 & 3.8961 & 3.7279 \\
\hline & 0.4 & 4.3696 & 4.4827 & 4.4684 & 4.3158 & 4.1044 & 3.9201 & 4.4544 & 4.5568 & 4.4857 & 4.2661 & 4.0291 & 3.8480 \\
\hline & 0.6 & 4.6735 & 4.7621 & 4.6996 & 4.4820 & 4.2250 & 4.0216 & 4.7548 & 4.8275 & 4.6957 & 4.4058 & 4.1326 & 3.9462 \\
\hline & 0.8 & 4.9393 & 5.0220 & 4.9145 & 4.6227 & 4.3147 & 4.1062 & 5.0157 & 5.0807 & 4.8851 & 4.5168 & 4.2091 & 4.0277 \\
\hline & 1 & 5.1084 & 5.2528 & 5.1153 & 4.7319 & 4.3824 & 4.1778 & 5.1872 & 5.3118 & 5.0573 & 4.5869 & 4.2734 & 4.0962 \\
\hline \multirow{6}{*}{0.6} & 0 & 3.5168 & 3.6060 & 3.5974 & 3.4976 & 3.3618 & 3.2656 & 3.5878 & 3.6667 & 3.6179 & 3.4741 & 3.3256 & 3.2418 \\
\hline & 0.2 & 3.8544 & 3.9154 & 3.8603 & 3.7045 & 3.5382 & 3.4420 & 3.9277 & 3.9716 & 3.8616 & 3.6582 & 3.4971 & 3.4138 \\
\hline & 0.4 & 4.1743 & 4.2038 & 4.0911 & 3.8659 & 3.6855 & 3.5879 & 4.2487 & 4.2520 & 4.0702 & 3.8004 & 3.6391 & 3.5547 \\
\hline & 0.6 & 4.4802 & 4.4773 & 4.2954 & 3.9930 & 3.8102 & 3.7102 & 4.5527 & 4.5150 & 4.2438 & 3.9207 & 3.7581 & 3.6719 \\
\hline & 0.8 & 4.7666 & 4.7407 & 4.4657 & 4.1026 & 3.9168 & 3.8140 & 4.8364 & 4.7665 & 4.3608 & 4.0235 & 3.8590 & 3.7707 \\
\hline & 1 & 5.0041 & 4.9964 & 4.5739 & 4.1980 & 4.0088 & 3.9030 & 5.0720 & 5.0103 & 4.4526 & 4.1122 & 3.9456 & 3.8548 \\
\hline \multirow{6}{*}{0.8} & 0 & 3.3114 & 3.3248 & 3.2222 & 3.0972 & 3.0468 & 3.0330 & 3.3727 & 3.3604 & 3.2036 & 3.0871 & 3.0437 & 3.0352 \\
\hline & 0.2 & 3.6434 & 3.6159 & 3.4296 & 3.2948 & 3.2383 & 3.2195 & 3.7076 & 3.6433 & 3.4033 & 3.2784 & 3.2295 & 3.2162 \\
\hline & 0.4 & 3.9614 & 3.8852 & 3.6097 & 3.4638 & 3.4000 & 3.3754 & 4.0273 & 3.8964 & 3.5750 & 3.4404 & 3.3848 & 3.3661 \\
\hline & 0.6 & 4.272 & 4.1327 & 3.7682 & 3.6099 & 3.5383 & 3.5073 & 4.3373 & 4.1120 & 3.7244 & 3.5791 & 3.5166 & 3.4921 \\
\hline & 0.8 & 4.5747 & 4.3398 & 3.9088 & 3.7375 & 3.6578 & 3.6204 & 4.6382 & 4.2705 & 3.8554 & 3.6992 & 3.6297 & 3.5994 \\
\hline & 1 & 4.859 & 4.5008 & 4.0345 & 3.8498 & 3.7619 & 3.7182 & 4.9191 & 4.4152 & 3.9713 & 3.8041 & 3.7275 & 3.6916 \\
\hline \multirow{6}{*}{1} & 0 & 2.979 & 2.8047 & 2.7842 & 2.8008 & 2.8328 & 2.8736 & 3.0293 & 2.8228 & 2.8012 & 2.8187 & 2.8519 & 2.8936 \\
\hline & 0.2 & 3.3064 & 3.0499 & 3.0047 & 3.0081 & 3.0310 & 3.0641 & 3.3601 & 3.0610 & 3.0149 & 3.0196 & 3.0438 & 3.0779 \\
\hline & 0.4 & 3.6243 & 3.2707 & 3.1977 & 3.1867 & 3.1995 & 3.2243 & 3.6802 & 3.2733 & 3.2001 & 3.1910 & 3.2056 & 3.2317 \\
\hline & 0.6 & 3.9402 & 3.4719 & 3.3687 & 3.3423 & 3.3446 & 3.3610 & 3.9970 & 3.4646 & 3.3626 & 3.3392 & 3.3439 & 3.3620 \\
\hline & 0.8 & 4.2622 & 3.6570 & 3.5215 & 3.4792 & 3.4710 & 3.4790 & 4.3179 & 3.6386 & 3.5064 & 3.4685 & 3.4634 & 3.4737 \\
\hline & 1 & 4.6011 & 3.8288 & 3.6590 & 3.6007 & 3.5819 & 3.5818 & 4.6526 & 3.7980 & 3.6347 & 3.5823 & 3.5677 & 3.5705 \\
\hline
\end{tabular}


Table 1. contd...

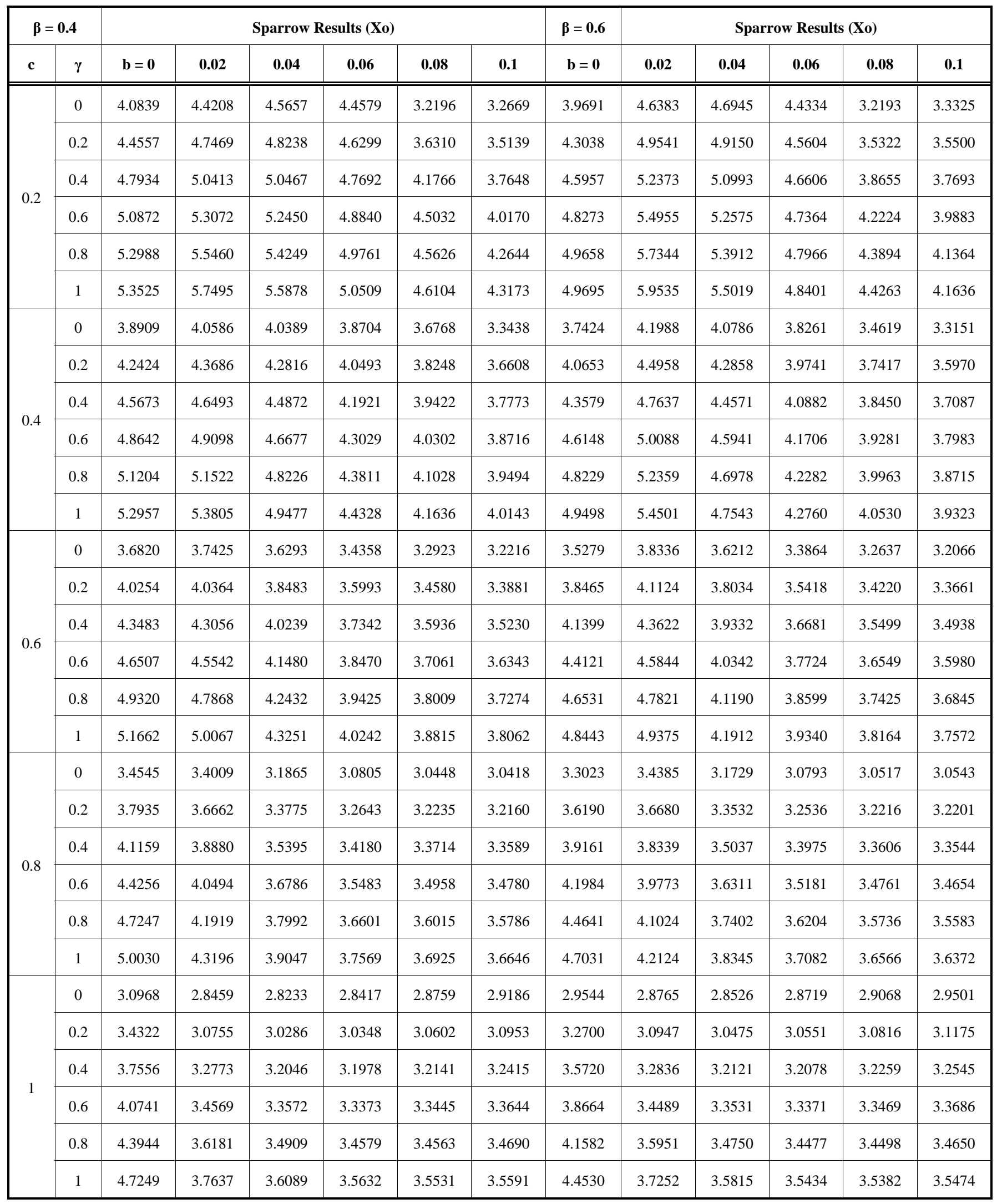




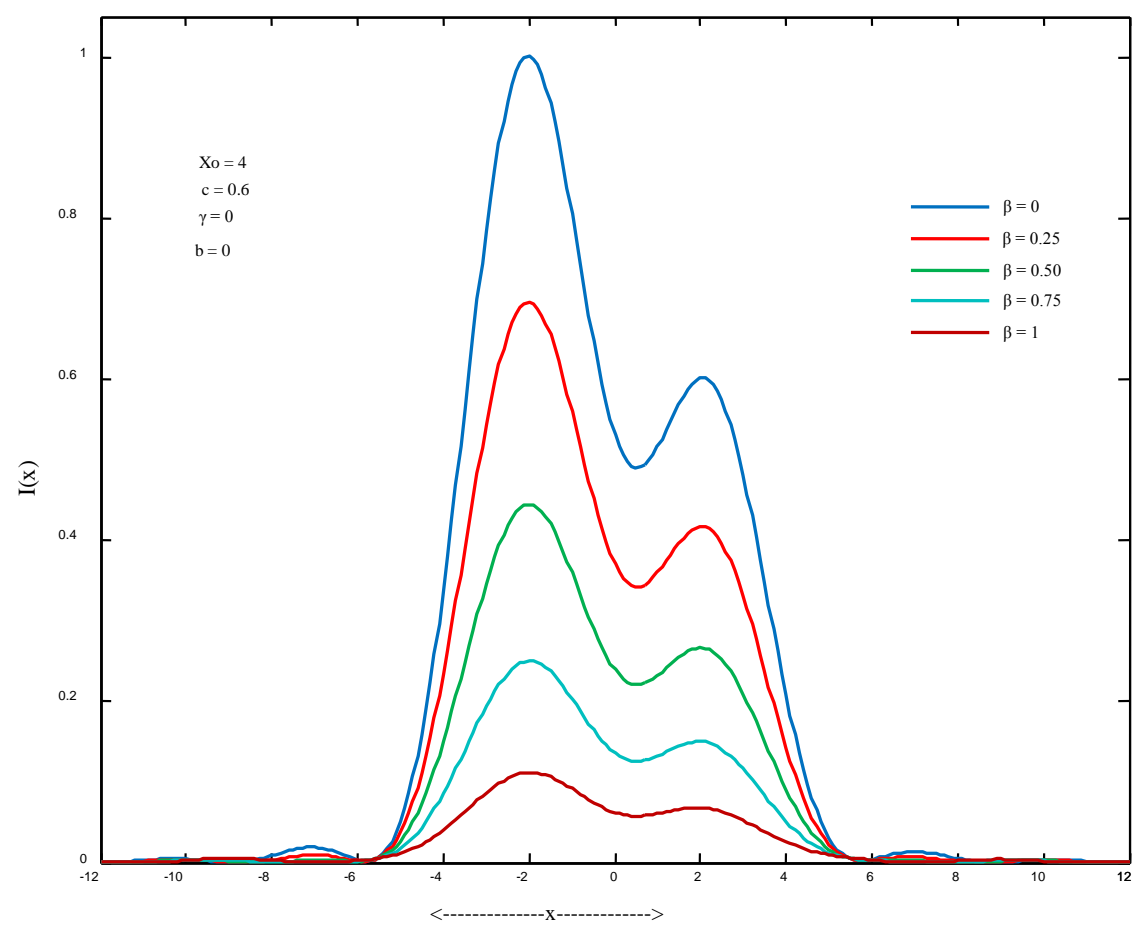

Fig. (3). Composite image intensity distribution for various values of $\beta$ under incoherent illumination.

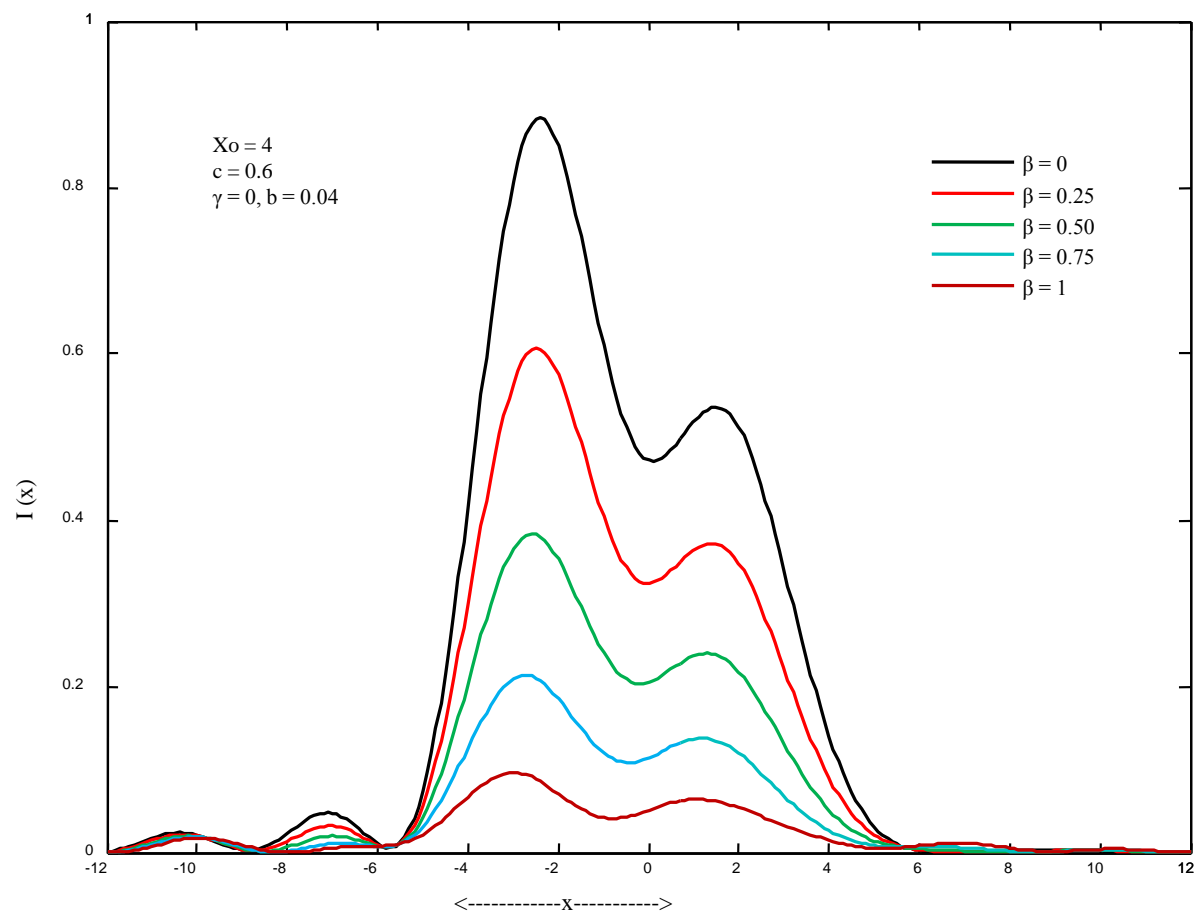

Fig. (4). Composite image intensity distribution for various values of $\beta$ under incoherent illumination for certain width of semicircular edge ring element $b=0.04$.

3.5974, 3.6179, 3.6293 and 3.6212 (from Table 1), respectively. As amplitude apodization controlling parameter held constant $(\beta=0.2$ or 0.6 ), the effect of asymmetric apodization (functions of $b$ value) in the resolution of two point objects of different intensity is illustrated in Figs. (5a) and (b). Asymmetric apodization found to be more effective in improving the resolution of optical imaging system. This study is found that left half axis intensity profile become broadened and shifted while on right half axis intensity profile becomes narrower. This observation improves with degree of asymmetric apodization for any value of $\beta$. Shift in the resultant intensity pattern can also be observed with respect to dip position. This effect is an evidence for superresolution phenomena. 


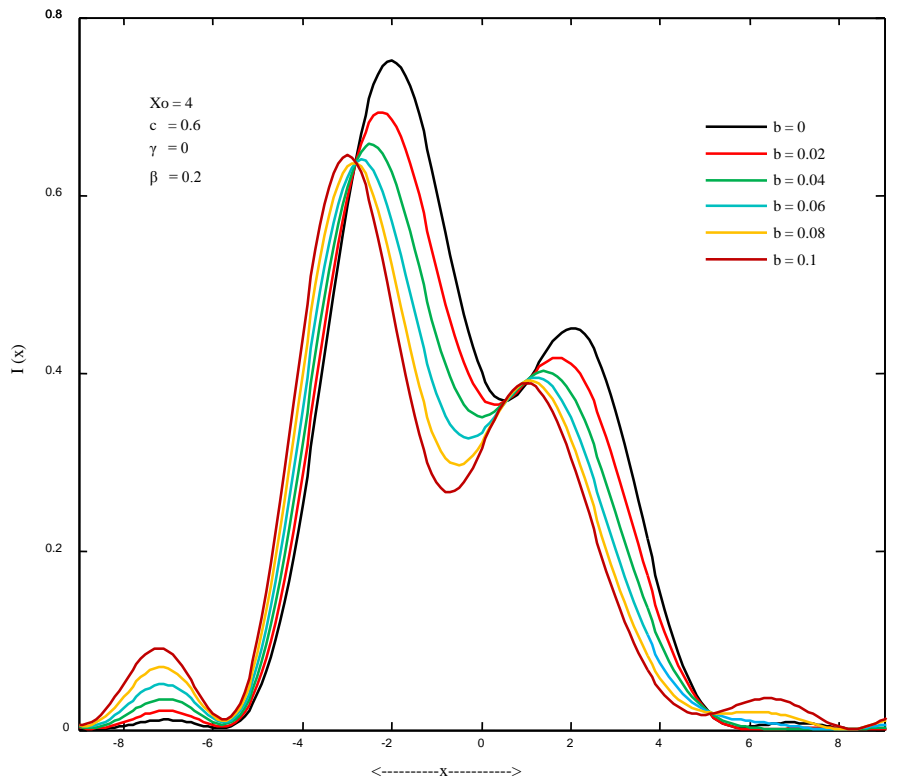

(a)

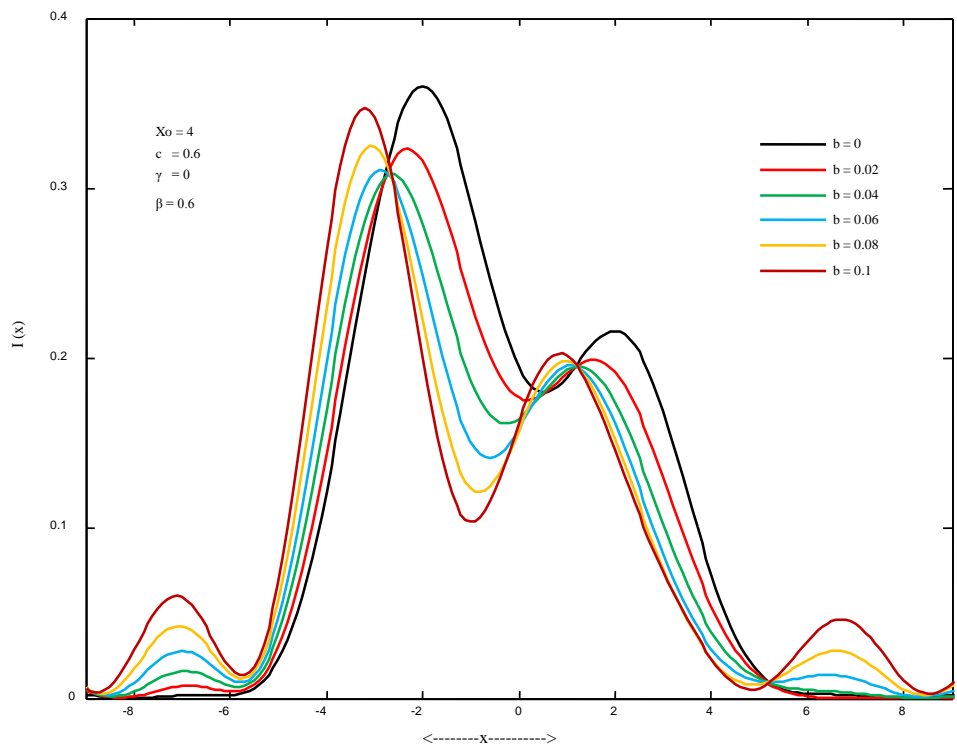

(b)

Fig. (5). Composite image intensity distribution for various values of ' $b$ ' under incoherent illumination in the presence of amplitude apodization: (a) $\beta=0.2$ (b) $\beta=0.6$.

Fig. (6) shows the intensity distribution as a function of $\mathrm{x}$ for various separations of two points of different intensity in the object plane under incoherent illumination $\gamma=0$. Here, the degree of asymmetric apodization is $b=0.1$ and the degree of amplitude apodization is $\beta=0.2$. For $\mathrm{Xo}=3$, there result a dip appears in the resultant intensity pattern. With the increase of Xo, the position of central dip moves toward low intensity maximum rendering an improvement in the resolution. Fig. (7) shows the intensity distribution in incoherent composite image of two point sources at Xo $=4$ for various values of the intensity ratio $\mathrm{c}$. In this figure in the case of two equally bright point sources $(c=1)$, marked resolution can be observed than that of rest of the conditions. It implies that three zone apodizer is effective in resolving two object points of equal intensity. The intensity distribution curves in Fig. (8) are plotted as function of $\mathrm{x}$ for various combinations of degree of coherence $\gamma$. The dependence of the sparrow resolution limits on apodization parameters $(b=$ 0.1 and $\beta=0.4$ ) is depicted. This figure illustrates that for $\mathrm{c}$ $=0.6$ at Xo $=4$, the dip appears on composite image intensity distribution of two object points under coherence illumination. As the coherence condition of light approaches the incoherent limit $(\gamma=0)$ the dip of intensity increases almost linearly with $\gamma$ values. This effect states that the influence of three zone pupil filter is found to be significant in improving the resolution of two distinct point sources of different intensities. 


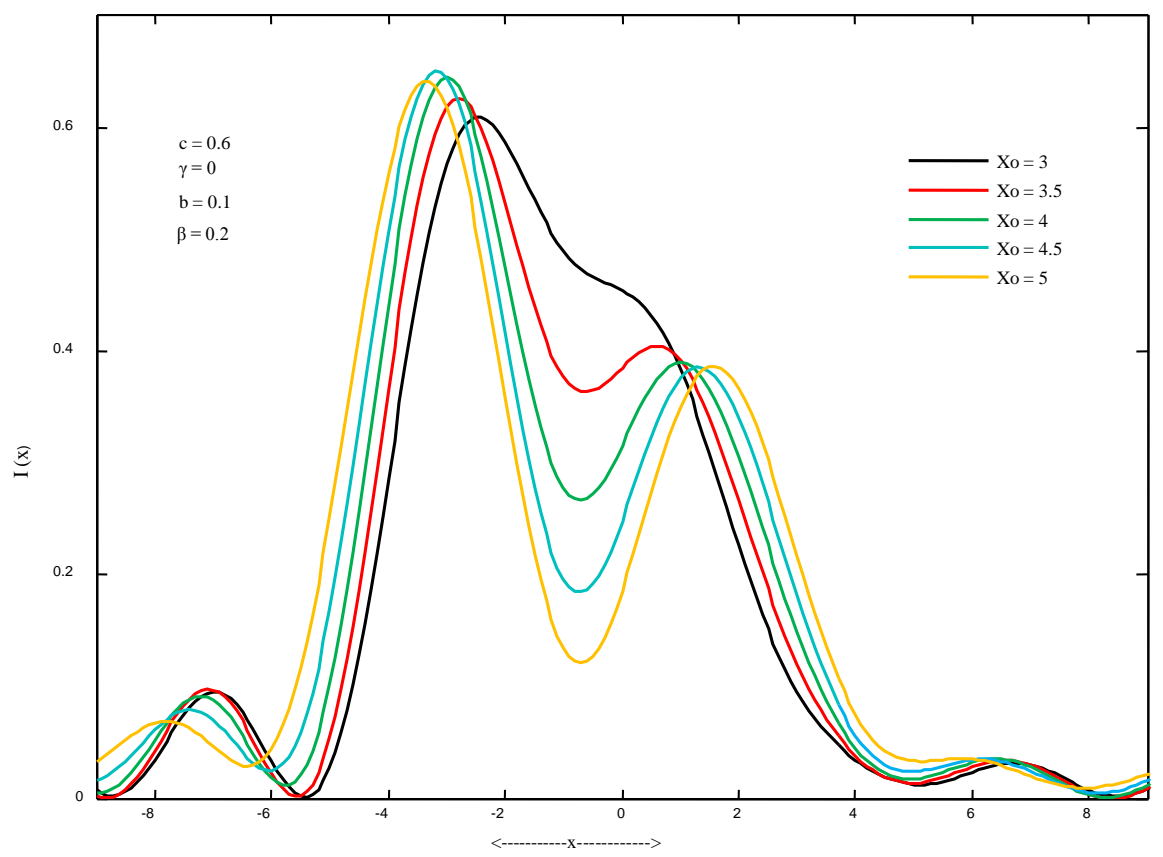

Fig. (6). Composite image intensity distribution for various values of point separation (Xo) under incoherent illumination with amplitude ( $\beta$ $=0.2)$ and phase $(\mathrm{b}=0.1)$ apodization.

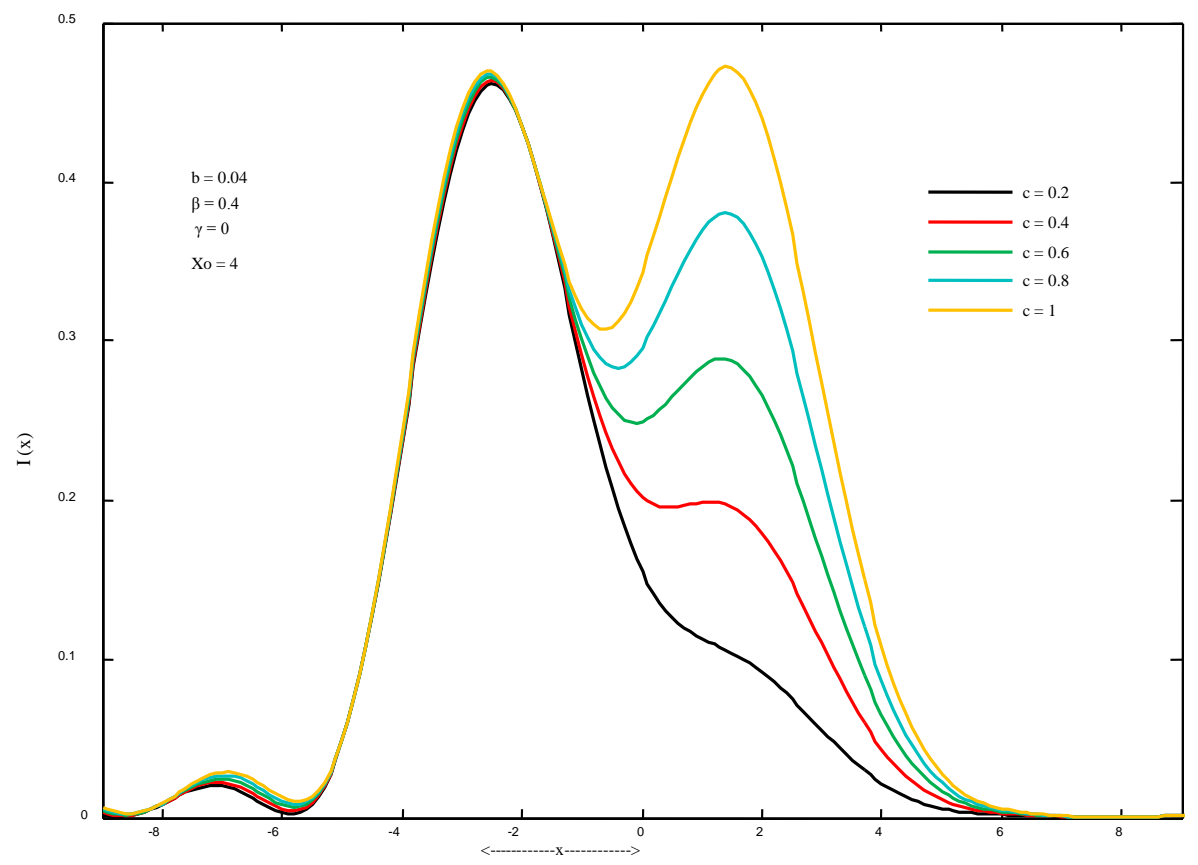

Fig. (7). Composite image intensity distribution for various values of 'c' under incoherent illumination with amplitude $(\beta=0.4)$ and phase (b $=0.04)$ apodization.

\section{CONCLUSION}

By employing amplitude and phase filter the Two-point resolution of optical imaging systems in partially coherent or coherent light has been studied in terms of modified Sparrow criterion. Present studies conclude that the resolution of composite image of two distinct object points is very much influenced by the degree of apodization. This influence resembles variedly with amplitude apodization $(\beta)$ and asymmetric apodization (b) on the resultant image intensity distribution. The present study is found to be more effective for certain values of $b(0.04,0.8$ and 0.1$)$ and $\beta(0.2,0.4$ and 0.6 ). This apodizer can be realized in practice with proper composition of dielectric coating materials by making use of standard fabrication techniques, this can be taken as future scope of present study. 


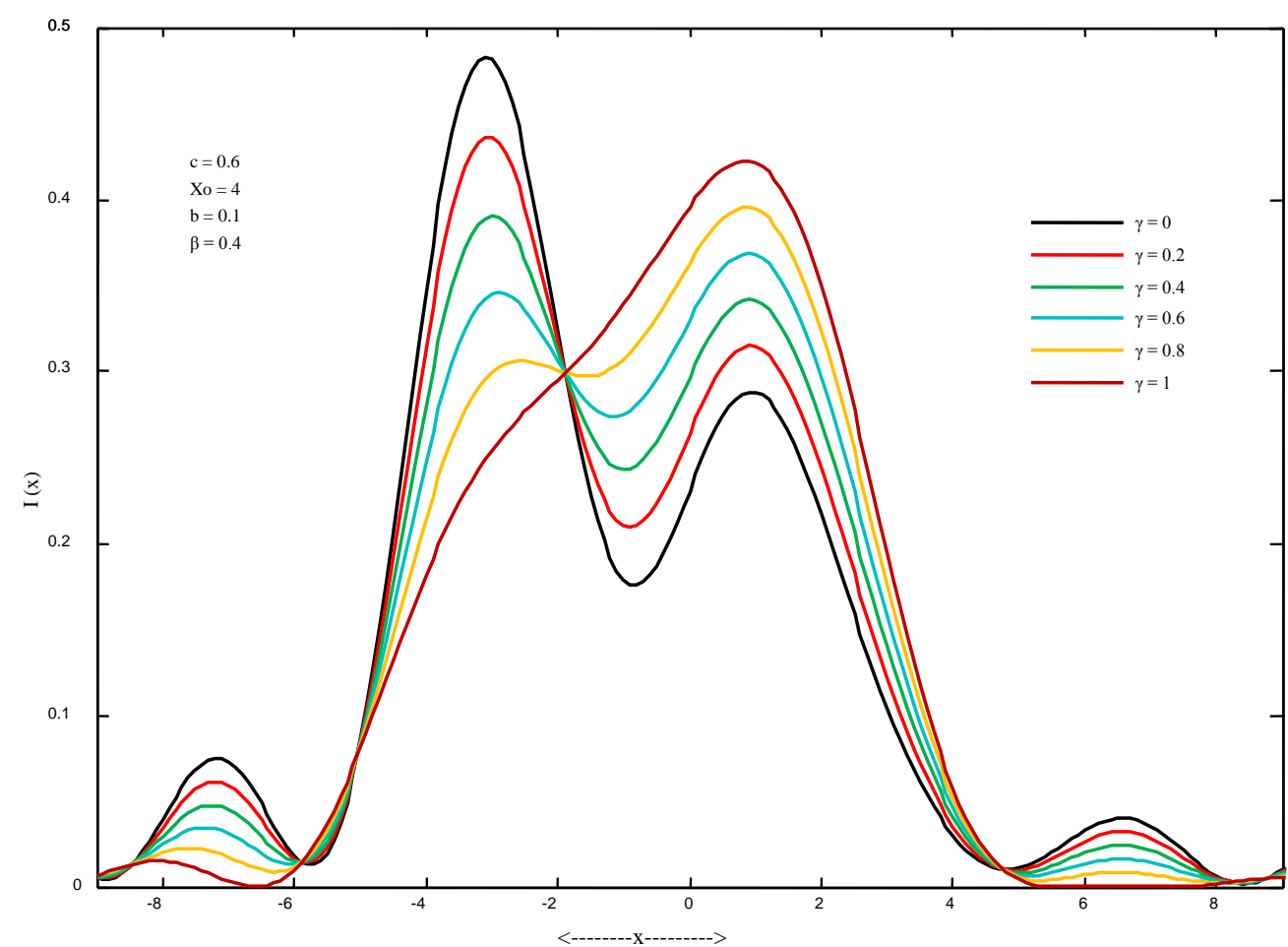

Fig. (8). Composite image intensity distribution under different coherence conditions of illumination with amplitude $(\beta=0.4)$ and phase apodization $(\mathrm{b}=0.1)$.

\section{CONFLICT OF INTEREST}

The author confirms that this article content has no conflict of interest.

\section{ACKNOWLEDGEMENTS}

Declared None.

\section{REFERENCES}

[1] Asakura T. Resolution of two unequally bright points with partially coherent light. Nouv Rev Opt 1974; 5: 169-77.

[2] Asakura T, Ueno T. Apodization for increasing two-point resolution by the sparrow criterion under the partially coherent illumination. Nouv Rev Opt 1974; 5: 349-59.

[3] Barakat R. Application of apodization to increase two-point resolution by Sparrow criterion. I-coherent illumination. J Opt Soc Am 1962; 52: 276-83.

[4] Ronchi V. Resolving power of calculated and detected images. J Opt Soc Am 1961; 51: 458-60.

[5] Nayyar VP, Verma NK. Two-point resolution of a Gaussian aperture operating in partially coherent light using various resolution criteria. Appl Opt 1978; 17: 2176-80.

[6] Osterberg H, Wissler F. The resolution of two particles in a bright field by coated microscope objectives. J Opt Soc Am 1949; 39: 558-66.

[7] Barakat R, Levin E. Application of apodization to increase twopoint resolution by the Sparrow criterion. II. Incoherent illumination. J Opt Soc Am 1963; 53: 274-82.

[8] Harris JL. Diffraction and resolving power. J Opt Soc Am 1964; 54: 931-6.

[9] di Francia GT. Resolving power and information. J Opt Soc Am 1955; 45: 497-501.
[10] Helstrom CW. The detection and resolution of optical signals. IEEE Trans Inf Theory 1964; IT-10: 275-87.

[11] Helstrom CW. Detection and resolution of incoherent objects by a background-limited optical system. J Opt Soc Am 1969; 59: 16475.

[12] Helstrom CW. Resolvability of objects from the standpoint of statistical parameter estimation. J Opt Soc Am 1970; 60: 659-66.

[13] Orhaug T. On the resolution of imaging systems. Opt Acta 1969; 16: 75-84.

[14] den Dekker AJ. Model-based optical two-point resolution. In: proceedings of icassp'96, ieee international conference on acoustics, speech, and signal processing. Institute of Electrical and Electronics Engineers, New York 1996; pp. 2395-8.

[15] den Dekker AJ. On two point resolution of imaging systems. Period Polytech ser El Eng 1997; 41: 167-83.

[16] den Dekker AJ, van den Bos A. Resolution: Survey. J Opt Soc Am A 1997; 1: 547-56.

[17] Reddy ANK, Sagar DK. Two point resolution of asymmetrically apodized optical systems. Opt Pura Apl 2013; 46: 215-22.

[18] Strutt JW. (Baron Rayleigh) Investigations in optics, with special reference to the spectroscope in Scientific Papers Press, Cambridge: Cambridge University Press 1899; 1: pp. 416-59.

[19] Sparrow CM. On spectroscopic resolving power. Astrophys J 1916; 44: 76-86.

[20] Sagar DK, Sayanna R, Goud MK, Goud SL. Effect of defocusing on the sparrow limits for apodized optical systems. Opt Commun 2003; 217: 59-67.

[21] Hopkins HH, Barham PM. The influence of the condenser on microscopic resolution. Proc Phy Soc B 1950; 63: 737-44.

[22] Cheng L, Siu GG. Asymmetric apodization. Meas Sci Technol 1991; 2: 198-202.

[23] Siu GG, Cheng L, Chiu DS, Chang KS. Improved side-lobe suppression in asymmetric apodization. J Phys D Appl Phys 1997; 27: 459-63. 
[24] Kowalczyk M, Zapata-Rodriguez CJ, Martinez-Corral M. Asymmetric apodization in confocal scanning systems. Appl Opt 1998; 37: 8206-14.

[25] Cichocki T, Martinez-Corral M, Kowalczyk M, Andres P. Annular binary filters for controlling the axial behaviour of optical systems. J Mod Opt 1998; 45: 227-37.
[26] Andres P, Martinez-Coral M, Ojeda-Casteneda J. Off-axis focal shift for rotationally non symmetric screens. Opt Lett 1993; 8: 1290-2.

[27] Yang W, Kostinski AB. One-sided achromatic phase apodization for imaging of extra solar planets. Astrophys J 2004; 605: 892-901.

Received: November 06, 2014

Revised: February 16, 2015

Accepted: April 06, 2015

(c) Reddy and Sagar; Licensee Bentham Open.

This is an open access article licensed under the terms of the Creative Commons Attribution Non-Commercial License (http://creativecommons.org/licenses/by$\mathrm{nc} / 3.0 /$ ) which permits unrestricted, non-commercial use, distribution and reproduction in any medium, provided the work is properly cited. 